\title{
Analysis of a relaxation process caused by the load variation adapted on the basis of second order electrical model
}

\author{
G. Nikishina, E. Denisov ${ }^{1}$ \\ Kazan National Research Technical University named after A.N. Tupolev - KAI, Department \\ of Radioelectronics and Information \& Measuring Technique, Kazan, Russia \\ 1 - corresponding author: genia-denisov@yandex.ru \\ Guzel Nikishina 0000-0002-0287-3388 \\ Evgenii Denisov 0000-0001-9677-8375
}

\begin{abstract}
The relaxation process of lithium batteries caused by load variation is considered. It is shown that such processes have strong dependence on internal physical and chemical processes and battery technical conditions. Theoretical expressions of the relaxation process caused by a steplike load variation have been obtained for $1^{\text {st }}$ and $2^{\text {nd }}$ order equivalent electrical circuits. The experimental investigations show that the obtained models fit the real relaxation processes and the behavior of the identified parameters could be explained by specific features of physical and chemical processes within the lithium battery. It should be noted that the obtained results can be generalized for a different type of electrochemical power source. The proposed approach makes it possible to provide means for electrochemical power source characterization and diagnostic, the main advantages of which are good time localization of measurement procedures and inexpensive apparatus implementation.
\end{abstract}

\section{Keywords}

lithium battery, electrochemical power sources, diagnostics, battery management system, load variation, in situ method

\section{Declarations}

\section{Funding}

This work has been supported by the Agreement No. 075-03-2020-051/6 (No. project fzsu2020-0020) funded by the Ministry of Science and Higher Education of the Russian Federation. 


\section{Introduction}

Currently lithium batteries (LB) are one of the most popular means for energy storage due to the ability to provide high power/capacity/energy density, stable operation, and small selfdischarging rate. However functional disadvantages of LB such as possibility of explosion due to overheating and battery short circuit, accelerated degradation related with overcharging/overdischarging or incorrect operation conditions are severe problems. Therefore, it is necessary to monitor battery technical state and detect degradation or critical operating modes. Toward this end many effective diagnostic tools have been developed. The corresponding tools are normally based on analysis of general electrical parameters [1-3] (stored charge, cell voltage/current, output power, etc.) or standard electrochemical characteristics (polarization curve, impedance, transient processes, etc.) [4-6]. The considering tools can be used to estimate complex parameters characterizing batteries technical state [7-11], such as State of Charge (SoC), State of Health $(\mathrm{SoH})$, State of Power (SoP). The diagnostic information obtained on the basis of analysis of the mentioned parameters and characteristics have wide area of application: input information for Battery Management Systems (BMS) [12-15], prognostic of technical characteristic, evaluation of available power, remaining useful life estimation, etc.

Every electrochemical system diagnostic method has different capabilities and the fields of applications. One of the approaches uses an analysis of polarization curve. It allows to evaluate parameters of charge transfer processes within the cell, namely losses related with diffusion, activation and ohmic polarization. This method has limited applicability due to technical difficulties, impossibility of on-line monitoring, and discharging processes within the batteries.

The promising direction of diagnostic method development is related with weak disturbances of the operating mode. The corresponding group of methods comprises electrochemical impedance spectroscopy (EIS) [16-19], current interrupt method [20], and time domain electrochemical impedance spectroscopy (TDEIS) [21-24]. EIS is intended to study reaction of the system to sinusoidal excitation signal, includes calculations of impedance and analyzes its amplitudes and phases variation in frequency domain. This approach is effectively used for investigation of internal processes occurring in electrochemical systems [25], their modeling [26], and diagnostics [27]. In spite of high informational content and reliability of different types of impedance spectroscopy diagnostic methods, they require expensive test equipment; long measurement time that makes application for battery diagnostics difficult due to instability of processes inside the most of electrochemical systems; low internal resistance of electrochemical power source (EPS) leads to requirements of high currents control. The internal resistance of EPS can be estimated by using current interrupt method. The main disadvantage of the method is relatively low information content (only one parameter is determined), but it has merits of localization in time and simplicity 
of the test equipment. The high information value of the impedance spectroscopy and good time localization of current interrupt method are combined within the time-domain electrochemical impedance spectroscopy.

Last decades a perspective method of fluctuation and noise diagnostics is developing [28-30]. The greater positive feature of this approach is the fact that it does not have any impact on the battery operation and makes diagnostics during its normal operation possible; however, they require technically complex and expensive measuring equipment as well as considerable time costs to gather the sufficient statistics. There are the numerous applicable demonstrations of fluctuation and noise diagnostics for EPS [31-32]. Above mentioned use different kinds of diagnostic features such as: statistical moments [33], power spectral density [34-36], wavelet coefficients [37-39], neural networks [40], etc. In spite of above said it can be claimed that a general approach for identification of informative features of electrical noises which allows building a diagnostic system for any type of batteries is absent.

The most widely used complex parameters which are $\mathrm{SoH}$ and $\mathrm{SoC}$ characterize the technical state of a battery. SoH is one of critical indicators for battery powered systems which estimates the degradation level of the batteries and is usually used for the battery condition monitoring. In recent years a numerous of different methods for $\mathrm{SoH}$ estimation has been developed [41-45] which can be classified as follows: trend analysis of parameters obtained from experimental data (i.e. EIS, as considered in [41]) and identification of model parameters [42]. The second group of methods is based on different methods using black box concept and models describing connection between input and output parameters of the system such as equivalent electrical circuit, various mathematical models, and neural networks. These methods in many cases involve machine learning techniques. For example, Ref. [43] describes the process of SoH monitoring for a high power lithium-ion cell based on recurrent neural networks to monitor and predict the aging processes. SoH can also be estimated basing on the energy measurement during charge and discharge processes in the normal conditions used for a battery [44]. Each method characterizing by a number of characteristics including estimation accuracy, test duration, feasibility.

SoC is a critical parameter that allows estimating of current technical condition of a battery. Traditionally SoC is defined as a ratio of available capacity to nominal one. It is obvious that SoC is a very important parameter and its monitoring helps to prevent overcharge and overdischarge, to improve the battery performance and to create a strategy for making battery application more efficient. Since complex chemical and physical processes within a battery as well as used modes of operation define battery operation, accurate estimation of the SoC becomes challenging. For that, there are lots of different approaches for its estimation, which are [45]: open circuit and 
terminal voltage methods; impedance spectroscopy; coulomb counting, neural network. As mentioned before, every of the existing methods has drawbacks limiting their field of application.

More specifically, analysis of literature shows that there is a gap of diagnostic techniques between expensive time-consuming methods that allow obtaining accurate complex information about the technical state of a battery and relatively simple methods providing the information about some specific parameters.

The gap is limiting possibilities to introduce diagnostic tools to many critical battery powered systems. To partially fill this gap, the present article proposes new diagnostic technique based on analysis of a relaxation process caused by the load variation. This technique uses advantages of time domain impedance spectroscopy concerning the good time localization and does not require powerful controllable current source. It makes possible to implement non expensive battery monitoring tools along with keeping high diagnostic capabilities. The obtained information is planned to be used for development of new battery diagnostic systems, estimation of complex parameters such as $\mathrm{SoC}, \mathrm{SoH}$, and as information source for perspective BMS.

\section{Methodology}

One of the most informative method for characterizing electrochemical power source is electrochemical impedance spectroscopy. Key idea which defines the theoretical basis of EIS consists in the fact that complex system under weak disturbances can be considered as a linear system. Therefore, the corresponding approaches such as linear impedance technique can be applied. In frame of this work, we propose to use small variation of an electrical load and analysis of corresponding responses to characterize electrochemical systems and their diagnostic features identification.

A linear equivalent electrical circuit is widely used in EIS to describe an electrochemical system under sufficiently small perturbation. At the same time, such linear circuit can be characterized by its time relaxation processes. Therefore, transient response is an evident tool for analyzing and diagnosing electrochemical systems.

Numerous interdependent physical and chemical processes such as ohmic polarization, diffusion and charge transfer inside electrochemical power source makes its relaxation processes to have complex transient behavior. As a rule, reaction on a small perturbation includes voltage step and relatively slow relaxations, which can be potentially used for in situ diagnostics [23]. The article [23] shows that the lithium batteries demonstrate the response on a step-like load current variation equal to the reaction of second order RC model (Fig. 2).

One of the conventional approach to analyze transient responses caused by a small perturbation is identification of equivalent model. Consider the corresponding identification could be 
implemented for the second order (Fig. 1) electrical model basing on analysis of the relaxation caused by a load resistance variation.

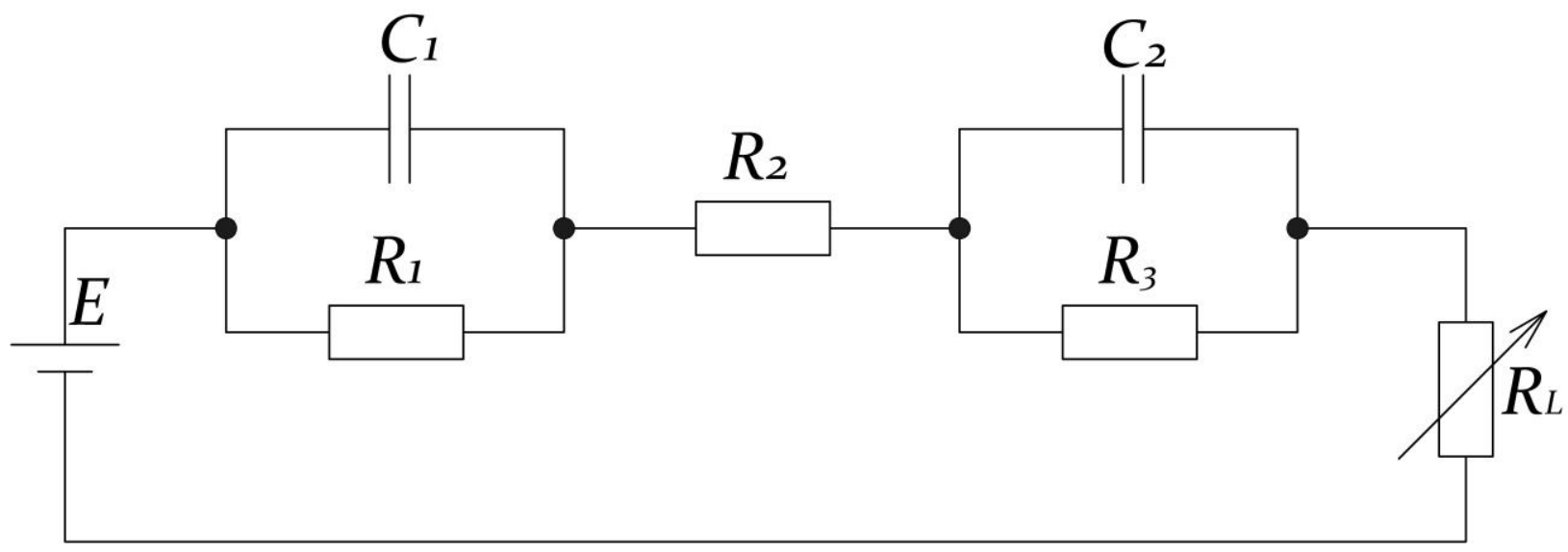

Fig. 1. The $2^{\text {nd }}$ order equivalent electrical circuit of the lithium battery for load variation induced relaxation process

The measurement procedure can be illustrated by Fig. 2, where one of the most effective way to obtain load variation, namely extra load $\Delta R$ commutation to the battery working with the main electrical load $R_{L 1}$, is shown. The commutation procedures evoke the transient responses for investigation.

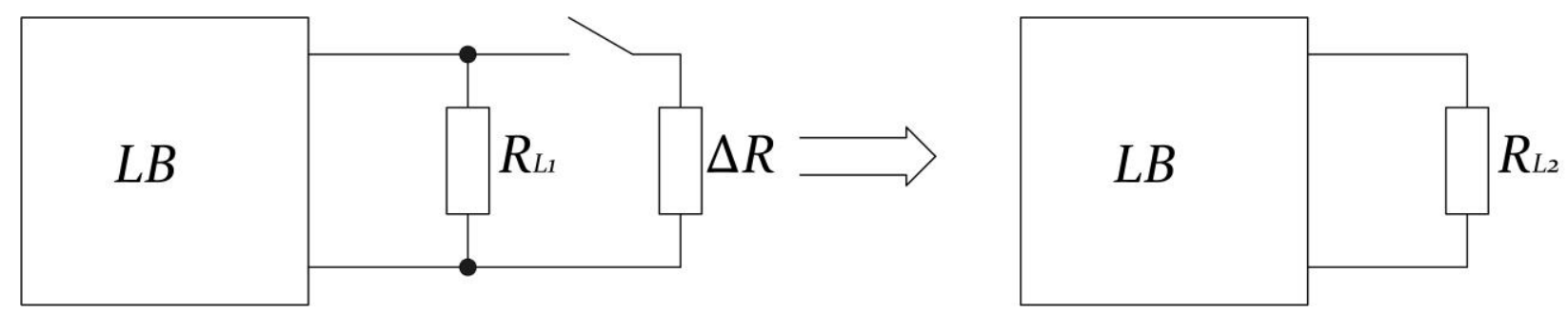

Fig. 2. The initiation of the load variation induced relaxation process

Consider the possibility to evaluate parameters of second order electrical model (Fig. 1) basing on the analysis of the considering relaxation processes. Basing on mesh current method, the circuit (Fig.2) can be described as:

$$
\left\{\begin{array}{c}
I_{11}\left(\frac{1}{p C_{1}}+R_{1}\right)-I_{22} \cdot R_{1}=-\frac{U_{C 10}}{p} ; \\
-I_{11} R_{1}+I_{22}\left(R_{1}+R_{2}+R_{3}+R_{L 2}\right)-I_{33} R_{3}=\frac{E}{p} \\
-I_{22} R_{3}+I_{33}\left(\frac{1}{p C_{2}}+R_{3}\right)=-\frac{U_{C 20}}{p} .
\end{array}\right.
$$

Equation system (1) allows us to find the Laplace image of current response on load variation:

$$
I_{22}=\frac{\left(R_{\Sigma 2}-R_{\Sigma 1}\right)\left(\left(C_{1} R_{1}^{2}+C_{2} R_{3}^{2}\right)+p C_{1} C_{2} R_{1} R_{3}\left(R_{1}+R_{3}\right)\right)}{R_{\Sigma 2}\left(1+p C_{1} R_{1}\right)\left(1+p C_{2} R_{3}\right)-p C_{1} R_{1}^{2}\left(1+p C_{2} R_{3}\right)-p C_{2} R_{3}^{2}\left(1+p C_{1} R_{1}\right)} \times
$$




$$
\times\left(-\frac{E}{R_{\Sigma 1} R_{\Sigma 2}}\right)+\frac{E}{p R_{\Sigma 1}},
$$

where

$$
\begin{aligned}
& R_{\Sigma 1}=R_{1}+R_{2}+R_{3}+R_{L 1}, \\
& R_{\Sigma 2}=R_{1}+R_{2}+R_{3}+R_{L 2},
\end{aligned}
$$

From general consideration, we could suppose that transient process of $2^{\text {nd }}$ order linear equivalent circuit has the following structure:

$$
U(t)=E-A e^{-B t}-C e^{-D t} ;
$$

It means that the Laplace image (2) had to presented in the following form:

$$
I_{22}=\frac{F}{p}-\frac{A}{p-B}-\frac{C}{p-D}=\frac{F}{p}-\frac{A p-A D+C p-B C}{p^{2}-B p-D p+B D} ;
$$

Comparing (2) and (6) we obtain the following equation system:

$$
\left\{\begin{array}{c}
A+C=C_{1} C_{2} R_{1} R_{2}\left(R_{1}+R_{3}\right)\left(R_{\Sigma 2}-R_{\Sigma 1}\right) \\
-A D-B C=\left(R_{\Sigma 2}-R_{\Sigma 1}\right)\left(C_{1} R_{1}^{2}+C_{2} R_{3}^{2}\right) ; \\
-B-C=R_{\Sigma 2} R_{\Sigma 1} \frac{C_{2} R_{3}\left(R_{\Sigma 2}-R_{3}\right)+C_{1} R_{1}\left(R_{\Sigma 2}-R_{1}\right)}{C_{1} C_{2} R_{1} R_{3}\left(R_{\Sigma 2}-R_{1}-R_{3}\right)} \\
B \cdot D=\frac{R_{\Sigma 1} R_{\Sigma 2}^{2}}{C_{1} C_{2} R_{1} R_{3}\left(R_{\Sigma 2}-R_{1}-R_{3}\right)} ; \\
F=\frac{E}{R_{\Sigma 1}} ;
\end{array}\right.
$$

Analysis of the equation system (7) shows the following. The parameters $A, B, C, D, F$ can be found from experimental data as a result of computer fitting procedures for the equation (5). The total resistances $R_{\Sigma 1}$ and $R_{\Sigma 2}$ are estimated on the basis of Ohm low in steady state conditions before and after commutation respectively. The last conclusion means that the equation system (7) comprising five unknown variables and only four equations since the last equation does not comprise any unknown variable. However, it is possible to extend the system by adding one of equations (15) or (16). In this case, we obtain the system of equations having a unique solution:

$$
\left\{\begin{array}{c}
A+C=C_{1} C_{2} R_{1} R_{2}\left(R_{1}+R_{3}\right)\left(R_{\Sigma 2}-R_{\Sigma 1}\right) \\
-A D-B C=\left(R_{\Sigma 2}-R_{\Sigma 1}\right)\left(C_{1} R_{1}^{2}+C_{2} R_{3}^{2}\right) \\
-B-C=R_{\Sigma 2} R_{\Sigma 1} \frac{C_{2} R_{3}\left(R_{\Sigma 2}-R_{3}\right)+C_{1} R_{1}\left(R_{\Sigma 2}-R_{1}\right)}{C_{1} C_{2} R_{1} R_{3}\left(R_{\Sigma 2}-R_{1}-R_{3}\right)} \\
B \cdot D=\frac{R_{\Sigma 1} R_{\Sigma 2}^{2}}{C_{1} C_{2} R_{1} R_{3}\left(R_{\Sigma 2}-R_{1}-R_{3}\right)} ; \\
R_{\Sigma 1}=R_{1}+R_{2}+R_{3}+R_{L 1}
\end{array}\right.
$$

The system (8) can be solved analytically or using numeric methods. Both approaches allow to find the parameters of electrical equivalent circuits. The proposed approach is applicable for any 
equivalent electrical circuits. It means that the approach based on analysis of an electrochemical system with variated electrical load can be adapted to extract information about their equivalent electrical circuit parameters.

Concluding the considered, the procedures for evaluation the electrical model parameter comprises the following steps:

1. Identification of the transient response model parameter (5) by means of computer fitting procedures

2. The electrical model parameters estimation on the basis of solving system (8).

The load resistance $R_{L 1}$ can be measured before start the procedure. For this purpose the current sensor can be introduced into measurement circuit.

\section{Numerical simulation}

The approach described above allows the equivalent electrical circuit parameters to be determined based on transient performance analysis. To validate the approach, a dedicated simulation of the EPS with a second-order equivalent electrical circuit (Fig. 1) was carried out in NI Multisim software. The model (Fig. 3) comprises elements of the equivalent electrical circuit resistances $R 1, R 2$, and $R 3$, and capacitances $C 1$ and $C 2$, as well as DC_POWER block $E$ corresponding to the open circuit potential of the EPS under test, and two load resistances $R L 1$ and $\triangle R$. The additional load is connected by the VOLTAGE_CONTROLLED_SPST switch S2, controlled by functional generator XFG1. The make-and-break status of switch S2 changes the load of the simulated EPS and starts the analyzed transient processes.

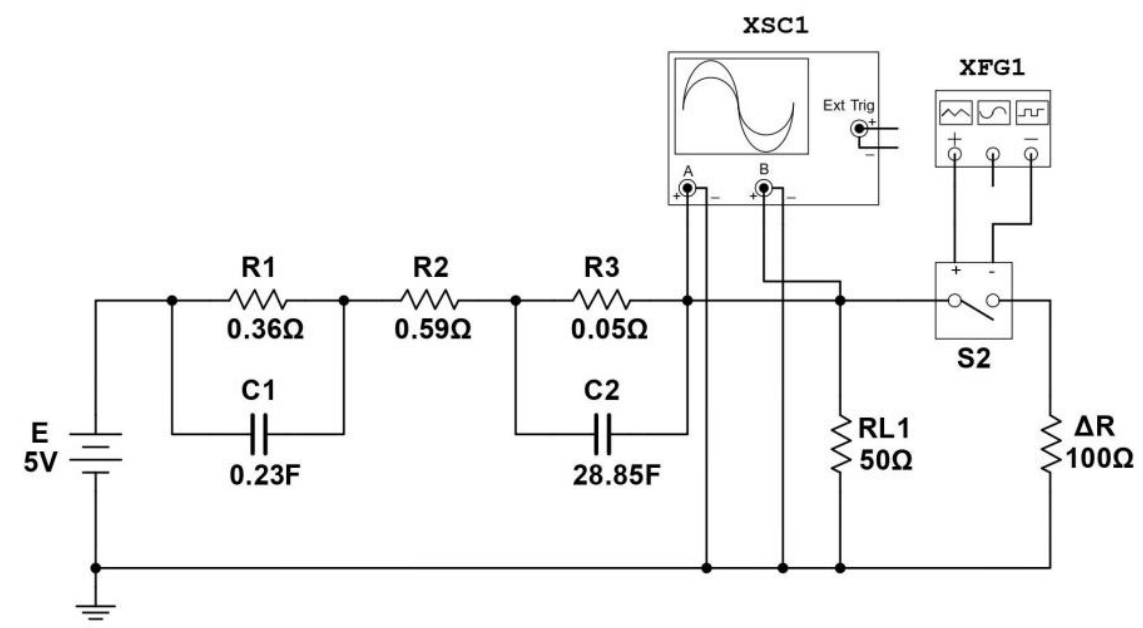

Fig. 3. NI Multisim simulation model for the generation of the transient response caused by load variation

As noted in the previous section, the requirements of the transient process are the following: (i) amplitudes do not take the EPS beyond the linear mode, and (ii) the experiment duration is long enough to estimate the transient process with sufficient accuracy. Within the present study, the 
parameters $R_{L 1}=50 \mathrm{Ohm}$ and $R_{L 2}=33.3 \mathrm{Ohm}$ are used to provide a linear mode of operation. The functional generator XFG1 operates with the following parameters: frequency of $0.5 \mathrm{~Hz}$, duty cycle of $50 \%$, amplitude of $5 \mathrm{~V}$, and "square wave" signal type.

Before the commutation, the load is equal to $50 \mathrm{Ohm}$; after commutation, it can be calculated as a parallel connection of $50 \mathrm{Ohm}$ and $100 \mathrm{Ohm}$ resistors, which is approximately equal to 33.3 Ohm. Hence, the model has a step-like load variation, which causes the required transient response. Said transient responses were recorded during half of the functional generator period of 10 seconds corresponding to the "on" position of the switch and processed in digital form.

In this way, the proposed control system generates an excitation signal to obtain the relaxation process caused by electrical load variation. Examples of the relaxation processes for different operational parameters are presented in Fig. 4. The experimental data have been normalized by subtracting the initial value and multiplying by -1 to improve visual representation.

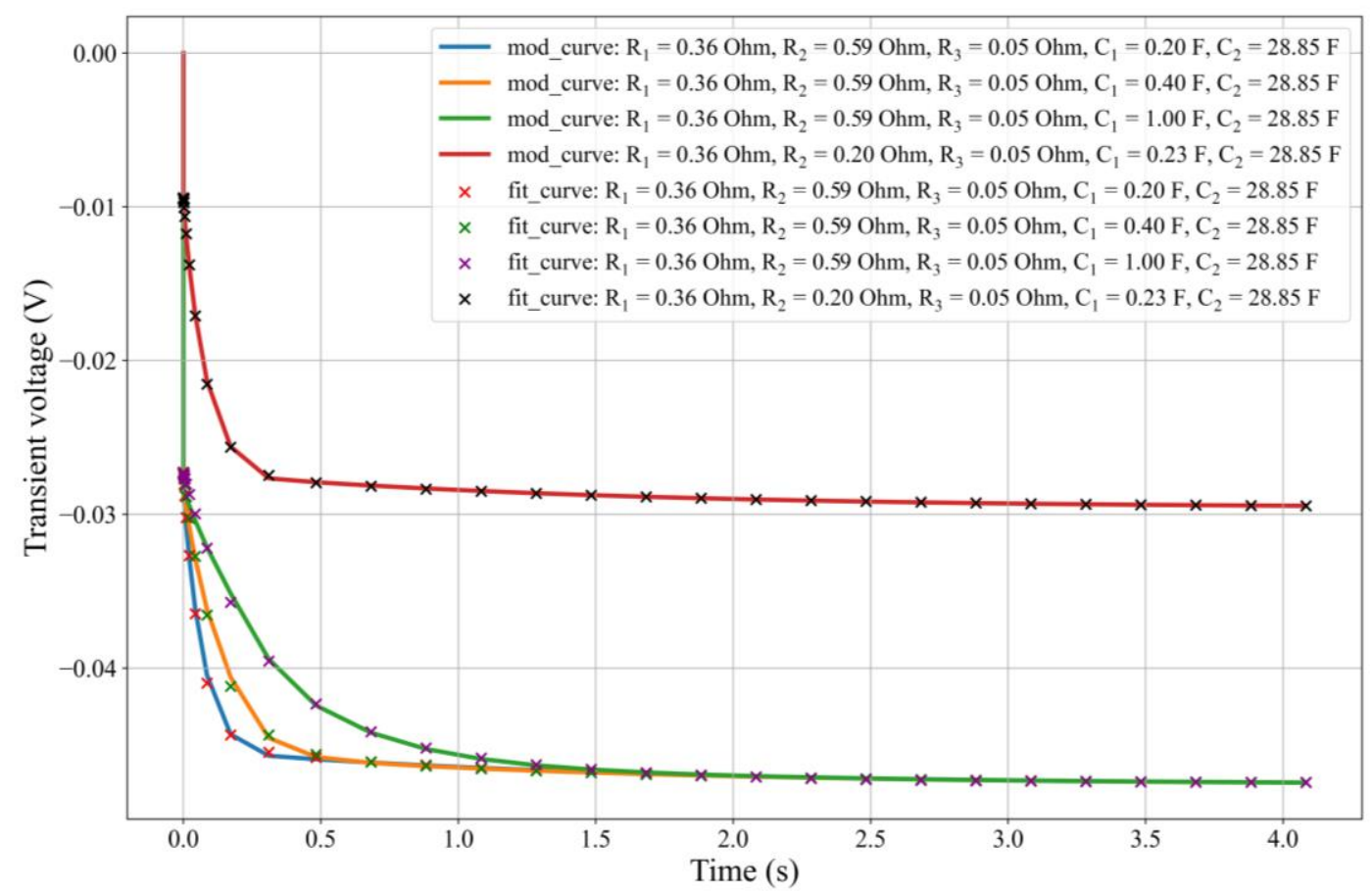

Fig. 4. Result of simulation and fitting for normalized relaxations of second-order equivalent electrical circuits caused by load variation

Analysis of the relaxation process caused by the load variation shows a strong dependence on the EPS model parameters, however, it has a similar structure, including the immediate step (not shown in Fig. 4) and subsequent relatively slow voltage relaxation, which confirms the conclusions of the previous sections. We shall now investigate the possibility of using these relaxations to estimate the parameters of the equivalent electrical circuit.

According to the algorithm presented above, the process of equivalent circuit parameter evaluation consists of two steps: computer fitting of model (10) and the solution of equation system 
(16). In this study, the computer fitting was implemented using Python programming language with the module scipy.optimize.curve.fit. More specifically the Levenberg-Marquardt algorithm was used to find the best-fit coefficients $U_{1}, U_{2}$, and $\tau$ of model (10). The results of the computer fitting are presented by the solid lines in Fig. 4. The small value of the obtained errors verifies the correctness of the model and allows us to use the results for further processing.

The fitted values are used to calculate the parameters of the equivalent circuit $R_{1}, R_{2}, R_{3}, C_{1}$, and $C_{2}$ by solving equation system (8). For the NI Multisim model with $R_{1}=0.36 \mathrm{Ohm}$, $R_{2}=0.59 \mathrm{Ohm}, R_{3}=0.05 \mathrm{Ohm}, C_{1}=0.23 \mathrm{~F}$, and $C_{2}=28.85 \mathrm{~F}$, the following parameters were estimated: $R_{1 \text { fitted }}=0.3617 \mathrm{Ohm}, R_{2 \text { fitted }}=0.5911 \mathrm{Ohm}, R_{3 \text { fitted }}=0.0471 \mathrm{Ohm}, C_{1 \text { fitted }}=0.2276 \mathrm{~F}$, and $C_{2 \text { fitted }}=29.9821 \mathrm{~F}$, corresponding to relative errors of $0.48 \%, 0.19 \%, 5.74 \%, 1.02 \%$, and $3.92 \%$, respectively.

To prove the reliability of the proposed method, the whole algorithm was performed for different values of $R_{1}, R_{2}, R_{3}, C_{1}$, and $C_{2}$ within the ranges $0.1-1.0 \mathrm{Ohm}, 0.1-1.0 \mathrm{Ohm}$, $0.03-1.00 \mathrm{Ohm}, 0.1-1.0 \mathrm{~F}$, and $10-40 \mathrm{~F}$, respectively. Analysis of the results shows that the average relative errors are $1.90 \%$ for $R_{1}, 0.03 \%$ for $R_{2}, 12.40 \%$ for $R_{3}, 1.24 \%$ for $C_{1}$, and $25.70 \%$ for $C_{2}$.

\section{Experimental investigation}

To check the proposed approach allowing determination of electrical equivalent circuit parameters the experimental investigations has been carried out.

\subsection{Experimental installation}

The experimental investigation of the proposed method has been carried out on the basis of the dedicated test installation. The commutation of an additional electrical load provides the excitation signal. Experimentally has been established that one of the best solutions to test the proposed method is to use electromagnetic relay since it introduces lower level of noises and interferences. The main disadvantage of this solution is relay chatter. However, it was found the relays without chatter for disconnection processes. Corresponding relay has been used to commutate $100 \mathrm{Ohm}$ extraload load $\Delta \mathrm{R}$ to the main load $R_{L 1}$ of $50 \mathrm{Ohm}$. The commutation process was controlled by microcontroller ATMEGA328P-PU. The test equipment comprises units to measure voltage and current generated by the EPS under test PXI Oscilloscope PXI-5922 of National Instruments.

\subsection{Experimental study}

The main goal of the experimental investigations is to study the relaxation processes of EPS induced by the step-like load variation and estimate their information content to identify parameters 
of equivalent electrical circuit. The obtained results allow to check the proposed models in accordance with experimental data, as well as to verify the proposed diagnostic methods. The EPS under study is Li-Ion unprotected battery Rexant with $2400 \mathrm{mAH}$ capacity at $3.7 \mathrm{~V}, 18650$ size. The experimental study has been conducted under low current operation mode defined by high load resistances (> $30 \mathrm{Ohm}$ ) to prevent effects of battery self-overheating. The step-like load variation between $33.3 \mathrm{Ohm}$ and $50 \mathrm{Ohm}$ has been carried out each 5 seconds. The examples of transient process measure for different states of charge are provided in Fig. 5.

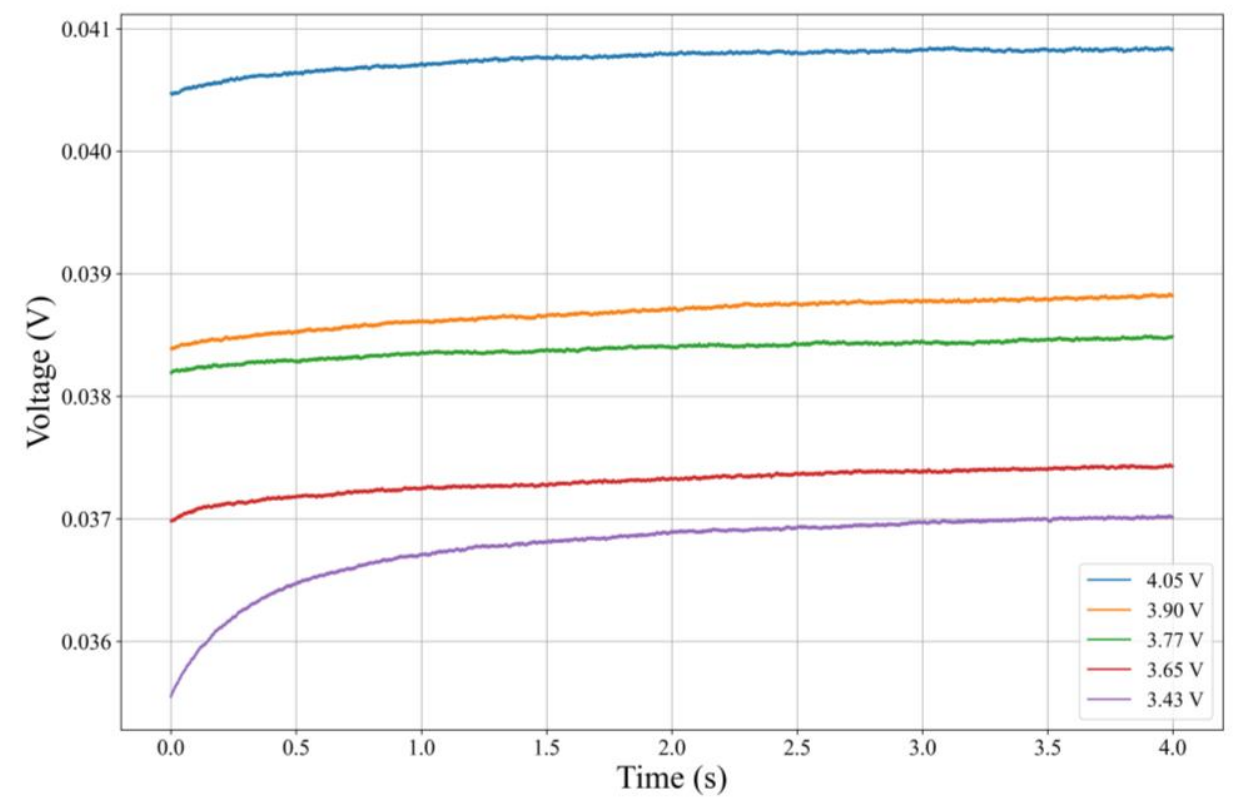

Fig. 5. The relaxation processes caused by the load variation for different open circuit potentials of the battery

Analysis of Fig. 5 shows that the relaxation processes has a strong dependence on battery voltage and the amplitudes grows with the battery discharging. The fitting process has been accomplished according to model (5) on the data, gathered by the experimental installation during the relay decoupling. The fitting process of real experimental data has some differences from one obtained during simulations, since we had to define start time of the transient process and define the opencircuit potential $\mathrm{E}$ of the battery. The start time has been defined by the dedicated synchronization system triggering the data acquisition. Since each transient response has been written in separate file, the fitting procedures have been applied to whole file. The first ten points have been ignored to exclude an impact of commutation noises on the results of analysis.

The evaluation of open circuit potential can be done based on analysis of the electrical equivalent circuit (Fig. 4) within steady state modes before and after relaxation processes correspondingly:

$$
\begin{aligned}
& E=\left(R_{x}-R_{L 1}\right) \cdot U_{L 1} / R_{L 1}, \\
& E=\left(R_{x}-R_{L 2}\right) \cdot U_{L 2} / R_{L 2},
\end{aligned}
$$


where $R_{x}$ is internal DC resistance of the battery, $R_{L 1}, R_{L 2}$ are load resistances, $U_{L 1}, U_{L 2}$ are steady state voltages before and after relaxation processes. Solving the equation system, we obtain the open-circuit potential:

$$
E=\left(\frac{U_{L 2}-U_{L 1}}{R_{L 2} U_{L 1}-R_{L 1} U_{L 2}} \cdot R_{L 1} R_{L 2}-R_{L 2}\right) \cdot U_{L 2} / R_{L 2},
$$

.Fig. 6 demonstrates the behavior of the equivalent electrical circuit parameters during discharging of the battery under test.
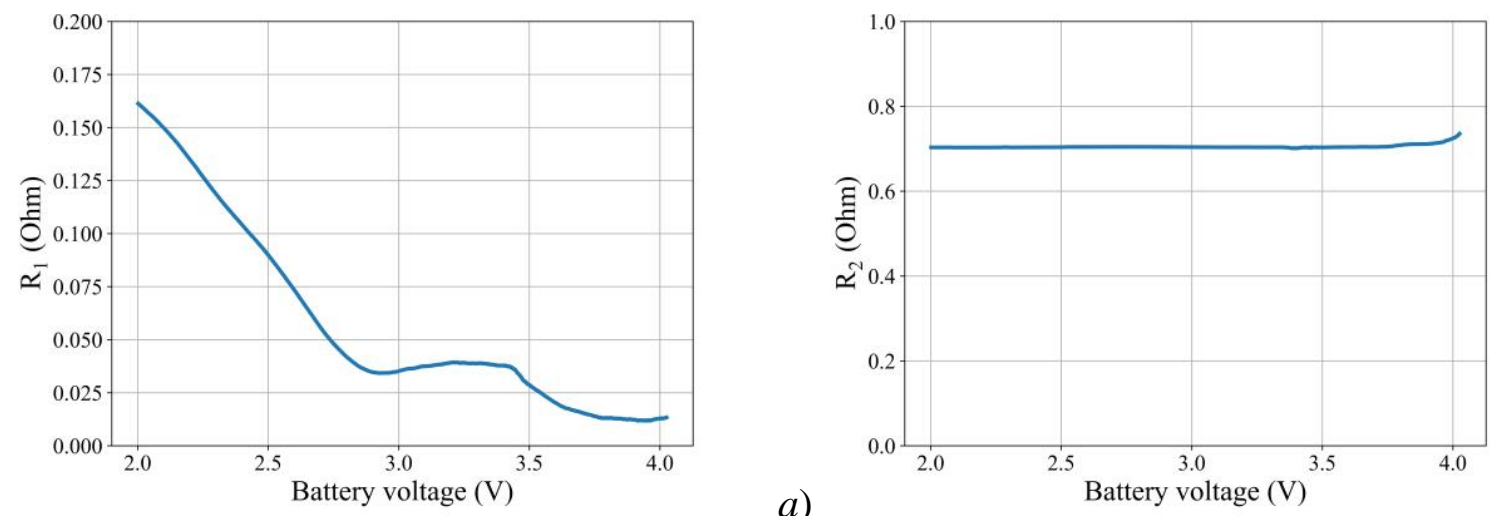

a)

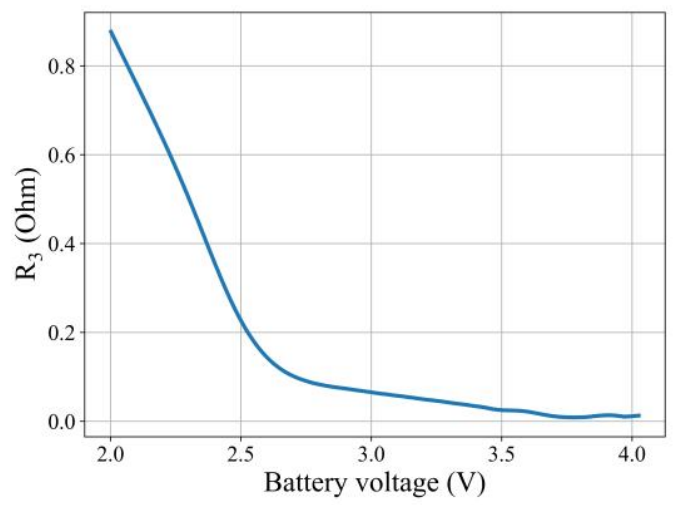

c)

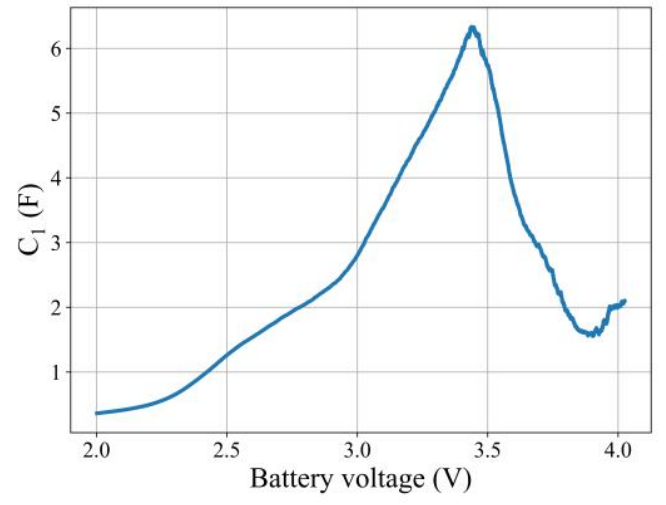

d)

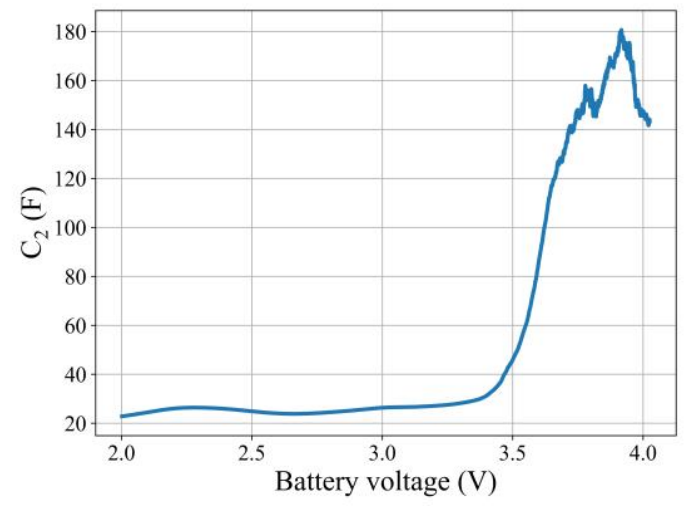

e)

Fig. 6. The dependencies of the second-order equivalent electrical circuit parameters $R_{1}(a), R_{2}$ $(b), R_{3}(c), C_{1}(d)$, and $C_{2}(e)$ on the battery voltage 
Analysis of the results presented in Fig. 6 shows that the resistances $R_{1}$ and $R_{3}$ monotonically increase during the battery discharge. The growth in these values accelerates when the battery voltage reaches values below 2.7-2.8 V. The described behavior can be explained by an increase in losses related to diffusion and charge transfer. The resistance $R_{2}$, related to ohmic polarization, stayed essentially constant across the considered range of open circuit potentials - this outcome is consistent with the findings of other researchers [46]. The higher capacitance $C_{2}$ demonstrates generally decreasing behavior with decreasing voltage, whereas, the capacitance $C_{1}$ has a maximum value near the nominal battery voltage and exhibits a monotonic decrease as the open-circuit voltage departs from the maximum point. This behavior of $C_{2}$ can be explained by the physical and chemical processes forming double layer capacity. The presence of the $C_{1}$ discharging curve peak near the battery voltage of $3.45 \mathrm{~V}$ should also be noted; at present, we have no explanation for this phenomenon, which will be investigated in future work.

The investigation of experimental results gives us possibility to suppose that the obtained results reflects internal processes within the battery and the obtained information could be used for characterization battery technical state and developing effective diagnostic means. The stated prepositions are also proved by a general ideas laying within the basis of the proposed approach, namely, the equivalence of impedance and time relaxation characteristics for a linear system as an example of which electrochemical systems can be considered in case of small external impact. The proposed approach has many advantages from practical point of view. Firstly, it has better localization in time in comparison with electrochemical impedance spectrometry. This advantage is especially important for electrochemical power sources due to impact on the results the discharging processes in case of a battery or temporal instability of the processes inside fuel cells. Furthermore, it significantly decreases the response time of diagnostic systems. Secondly, the proposed approach requires simpler and more inexpensive apparatus, which potentially allows manufacturer to embed monitoring means into the battery.

\section{Conclusion}

The relaxation processes caused by a load variation has been studied within lithium batteries. It follows from general considerations that relaxation processes of linear systems have the same information content as impedance spectra. Use of the relaxation processes gives a number of advantages such as better time localization of the measurement procedures and simpler measurement equipment. The relaxation processes caused by a current or potential variation are relatively simple from data processing point of view, however, still require powerful electronic means with control loops. Within the present article, the new method of electrochemical power source investigation based on analysis of relaxation process induced by a load variation is proposed. 
The main advantage of this approach is simple apparatus means using commutated additional load, or even natural variations of electrical load.

\section{Acknowledgments}

This work has been supported by the Agreement No. 075-03-2020-051/6 (No. project fzsu2020-0020) funded by the Ministry of Science and Higher Education of the Russian Federation.

\section{References}

[1] Lee S, Kim J, Lee J, Cho BH (2008) State-of-charge and capacity estimation of lithium-ion battery using a new open-circuit voltage versus state-of-charge. J Power Sources 185(2):13671373. https://doi.org/10.1016/j.jpowsour.2008.08.103.

[2] Zhang D, Haran BS, Durairajan A, White RE, Podrazhansky Y, Popov BN (2000) Studies on capacity fade of lithium-ion batteries. J Power Sources 91(2):122-129. https://doi.org/10.1016/S0378-7753(00)00469-9.

[3] Li H, Tang Y, Wang Z, Shi Z, Wu S, et al (2008) A review of water flooding issues in the proton exchange membrane fuel cell. J Power Sources 178:103-17. https://doi.org/10.1016/j.jpowsour.2007.12.068.

[4] Birkl CR, Roberts MR, McTurk E, Bruce PG, Howey DA (2017) Degradation diagnostics for lithium ion cells. J Power Sources 341:373-386. https://doi.org/10.1016/j.jpowsour.2016.12.011.

[5] Zalka D, Péter L (2020) On the evolution and application of the concept of electrochemical polarization. J Solid State Electrochem 24:2595-2602. https://doi.org/10.1007/s10008-020-046823.

[6] Nikishin TP, Denisov ES, Adiutantov NA (2020) Relaxation processes of solid polymer hydrogen fuel cells batteries and estimating of their diagnostic properties. Instruments and Systems: Monitoring, Control, and 7:1-12. https://doi.org/10.25791/pribor.07.2020.1187 (In Russian).

[7] Han X, Ouyang M, Lu L, Li J, Zheng Y, Li Z (2014) A comparative study of commercial lithium ion battery cycle life in electrical vehicle: Aging mechanism identification. J Power Sources 251:38-54. https://doi.org/10.1016/j.jpowsour.2013.11.029.

[8] Huet F (1998) A review of impedance measurements for determination of the state-ofcharge or state-of-health of secondary batteries. J Power Sources 70(1):59-69. https://doi:10.1016/S0378-7753(97)02665-7. 
[9] Charkhgard M, Farrokhi M (2010) State-of-Charge estimation for lithium-ion batteries using neural networks and EKF. IEEE Transactions on Industrial Electronics 57(12):4178-4187. https://doi:10.1109/TIE.2010.2043035.

[10] Wei C, Benosman M, Kim T (2019) Online parameter identification for state of power prediction of lithium-ion batteries in electric vehicles using extremum seeking. Int $\mathrm{J}$ Control Autom Syst 17:2906-2916. https://doi.org/10.1007/s12555-018-0506-y.

[11] Piller S, Perrin M, Jossen A (2001) Methods for state-of-charge determination and their applications. J Power Sources 96(1):113-120. https://doi.org/10.1016/S0378-7753(01)00560-2.

[12] Bian X, Liu L, Yan J (2019) A model for state-of-health estimation of lithium ion batteries based on charging profiles. Energy 177:57-65. https://doi.org/10.1016/j.energy.2019.04.070.

[13] Meissner B, Richter G (2003) Battery monitoring and electrical energy management: precondition for future vehicle electric power systems. J Power Sources 116(1-2):79-98. https://doi.org/10.1016/S0378-7753(02)00713-9.

[14] Cheng KWE, Divakar BP, Wu H, Ding K, Ho HF (2011) Battery-management system (BMS) and SOC development for electrical vehicles. IEEE Transactions on Vehicular Technology 60(1): 76-88. https://doi: 10.1109/TVT.2010.2089647.

[15] Zou Y, Hu X, Ma H, Li SE (2015) Combined State of Charge and State of Health estimation over lithium-ion battery cell cycle lifespan for electric vehicles. J Power Sources 273:793-803. https://doi.org/10.1016/j.jpowsour.2014.09.146.

[16] Bezmalinovic D, Simic B, Barbir F (2015) Characterization of PEM fuel cell degradation by polarization change curves. J Power Sources 294:82-87. https://doi.org/10.1016/j.jpowsour.2015.06.047.

[17] Love CT, Virji MBV, Rocheleau RE, Swider-Lyons KE (2014) State-of-health monitoring of $186504 \mathrm{~S}$ packs with a single-point impedance diagnostic. J Power Sources 266:512-519. https://doi.org/10.1016/j.jpowsour.2014.05.033.

[18] Tröltzsch U, Kanoun O, Tränkler H-R (2006) Characterizing aging effects of lithium ion batteries by impedance spectroscopy. Electrochimica Acta 51(8-9):1664-1672. https://doi.org/10.1016/j.electacta.2005.02.148.

[19] Mantia F, Vetter J, Novak P (2008) Impedance spectroscopy on porous materials: a general model and application to graphite electrodes of lithium-ion batteries. Electrochimica Acta 53(12):4109-4121. https://doi.org/10.1016/j.electacta.2007.12.060.

[20] Cooper KR, Smith M (2006) Electrical test methods for on-line fuel cell ohmic resistance measurement. J Power Sources 160:1088-1095. https://doi.org/10.1016/j.jpowsour.2006.02.086. 
[21] Boskoski P, Debenjak A, Boshkoska BM (2017). Fast electrochemical impedance spectroscopy as a statistical condition monitoring tool. SpringerBriefs in Applied Sciences and Technology, Springer. 83 p.

[22] Denisov E, Nigmatullin R, Evdokimov Y, Timergalina G (2018) Lithium battery transient response as a diagnostic tool. J Electron Mater 47(8):4493-4501. https://doi.org/10.1007/s11664018-6346-y.

[23] Denisov E, Nikishina G, Demidov A (2019) Automated excitation signal generation system for time-domain impedance spectroscopy. Ural Symposium on Biomedical Engineering, Radioelectronics and Information Technology (USBEREIT) 328-331. https://doi.org/10.1109/USBEREIT.2019.8736564.

[24] Denisov E, Nikishina G, Demidov A (2020) Electrochemical power sources relaxations related with step-like load variation. Ural Symposium on Biomedical Engineering, Radioelectronics and Information Technology (USBEREIT) 230-233. https://doi.org/10.1109/USBEREIT48449.2020.9117665.

[25] He YB, Li B, Yang QH. et al. (2011) Effects of current densities on the formation of LiCoO2/graphite lithium ion battery. J Solid State Electrochem 15:1977-1985. https://doi.org/10.1007/s10008-010-1220-8.

[26] Westerhoff U, Kroker T, Kurbach K, Kurrat M (2016) Electrochemical impedance spectroscopy based estimation of the state of charge of lithium-ion batteries. J Energy Storage 8:244-256. https://doi.org/10.1016/j.est.2016.09.001.

[27] Yuan X, Wang H, Sun JC, Zhang J (2007) AC impedance technique in PEM fuel cell diagnosis-A review. Int J Hydrog Energy 32(17):4365-4380. https://doi.org/10.1016/j.ijhydene.2007.05.036.

[28] Denisov ES, Evdokimov YuK, Martemianov S, Thomas A, Adiutantov N (2017) Electrochemical noise as a diagnostic tool for PEMFC. Fuel Cells 17:225-237. https://doi.org/10.1002/fuce.201600077.

[29] Astafev EA, Ukshe AE, Dobrovolsky YA (2018) Measurement of electrochemical noise of a Li/MnO2 primary lithium battery. J Solid State Electrochem 22:3597-3606. https://doi.org/10.1007/s10008-018-4074-0.

[30] Astafev EA (2020) The measurement of electrochemical noise of a Li-ion battery during charge-discharge cycling. Measurement 154:107492. https://doi.org/10.1016/j.measurement.2020.107492.

[31] Timergalina GV, Denisov ES (2018) Study of lithium battery fluctuations in the opencircuit conditions. 2018 International Conference on Actual Problems of Electron Devices Engineering (APEDE) 153-155. https://doi.org/10.1109/APEDE.2018.8542177. 
[32] Martemianov S, Adiutantov N, Evdokimov YK et al. (2015) New methodology of electrochemical noise analysis and applications for commercial Li-ion batteries. J Solid State Electrochem 19:2803-2810. https://doi.org/10.1007/s10008-015-2855-2.

[33] Maizia R, Dib A, Thomas A et al. (2018) Statistical short-time analysis of electrochemical noise generated within a proton exchange membrane fuel cell. J Solid State Electrochem 22:16491660. https://doi.org/10.1007/s10008-017-3848-0.

[34] Denisov ES (2008) Nonlinear and linear electric models of a hydrogen fuel cell and identification of it's parameters. Nonlinear world 6(8): 81-85 (In Russian).

[35] Martemianov S, Thomas A, Adiutantov N et al. (2020) Electrochemical noise analysis of a PEM fuel cell stack under long-time operation: noise signature in the frequency domain. J Solid State Electrochem 24:3059-3071. https://doi.org/10.1007/s10008-020-04759-z.

[36] Legros B, Thivel P-X, Bultel Y, Nogueira RP (2011) First results on PEMFC diagnosis by electrochemical noise. Electrochem Commun 13(12):1514-1516. https://doi.org/10.1016/j.elecom.2011.10.007.

[37] Pahon E, Hissel D, Jemei S, Yousfi-Steiner N (2016) Relative wavelet energy as a diagnosis tool for pem fuel cells. 2016 IEEE Vehicle Power and Propulsion Conference (VPPC) 1-6. https://doi.org/10.1109/VPPC.2016.7791760.

[38] Shindor OV, Denisov ES, Evdokimov YuK (2013) Investigation of the diagnostic capabilities of the wavelet transform for analyzing the electrical fluctuations of a hydrogen fuel cell. Bulletin of Kazan State Technical University named after A.N. Tupolev 4:120-124 (In Russian).

[39] Rubio MA, Bethune K, Urquia A, St-Pierre J (2016) Proton exchange membrane fuel cell failure mode early diagnosis with wavelet analysis of electrochemical noise. Int $\mathbf{J}$ Hydrogen Energy 41:14991-15001. https://doi.org/10.1016/j.ijhydene.2016.05.292.

[40] Vichard L, Harel F, Ravey A, Venet P, Hissel D (2020) Degradation prediction of PEM fuel cell based on artificial intelligence. Int J Hydrog Energy 45(29):14953-14963. https://doi.org/10.1016/j.ijhydene.2020.03.209.

[41] Li Y, Abdel-Monem M, Gopalakrishnan R et al. (2018) A quick on-line state of health estimation method for Li-ion battery with incremental capacity curves processed by Gaussian filter. J Power Sources 373:40-53. https://doi.org/10.1016/j.jpowsour.2017.10.092.

[42] Eddahech A, Briat O, Bertrand N, Delétage J-Y, Vinassa J-M (2012) Behavior and stateof-health monitoring of Li-ion batteries using impedance spectroscopy and recurrent neural networks. Int J Electr Power Energy Syst 42:487-494. https://doi.org/10.1016/j.ijepes.2012.04.050. 
[43] Camci F, Ozkur C, Toker O, Atamuradov V (2015) Sampling based State of Health estimation methodology for Li-ion Batteries. J Power Sources 278: 668-674. https://doi.org/10.1016/j.jpowsour.2014.12.119.

[44] Zhang J, Lee J (2011) A review on prognostics and health monitoring of Li-ion battery. J Power Sources 196(15):6007-6014. https://doi.org/10.1016/j.jpowsour.2011.03.101.

[45] Chang W-Y (2013) The State of Charge estimating methods for battery: a review. International Scholarly Research Notices 2013:1-7. https://doi.org/10.1155/2013/953792.

[46] W. Waag, S. Käbitz, D.U. Sauer, Experimental investigation of the lithium-ion battery impedance characteristic at various conditions and aging states and its influence on the application, Applied Energy. 102 (2013) 885-897. https://doi.org/10.1016/j.apenergy.2012.09.030 